\title{
VLBI Astrometry of Planetary Orbiters
}

\section{Dayton L. Jones ${ }^{1}$}

Jet Propulsion Laboratory, California Institute of Technology

Pasadena, CA, USA

E-mail: dayton.jones@jpI.nasa.gov

\section{Ed Fomalont}

National Radio Astronomy Observatory

Charlottesville, VA, USA

E-mail: efomalonenrao.edu

\section{Vivek Dhawan}

National Radio Astronomy Observatory

Socorro, NM, USA

E-mail: vdhawanenrao.edu

\section{Jon Romney}

National Radio Astronomy Observatory

Socorro, NM, USA

E-mail: jromney@nrao.edu

\section{William M. Folkner}

Jet Propulsion Laboratory, California Institute of Technology Pasadena, CA, USA

E-mail: William.M.Folknerajpl.nasa.gov

\section{Gabor E. Lanyi}

Jet Propulsion Laboratory, California Institute of Technology

Pasadena, CA, USA

E-mail: Gabor.E.Lanyiajpl.nasa.gov

\section{James S. Border}

Jet Propulsion Laboratory, California Institute of Technology

Pasadena, CA, USA

E-mail: James.S.BorderQjpl.nasa.gov

\section{Robert A. Jacobson}

Jet Propulsion Laboratory, California Institute of Technology Pasadena, CA, USA

E-mail: Robert.A. Jabobsonejpl nasa.gov

\section{$1 \quad$ Speaker}


Multi-epoch VLBA observations of the Cassini spacecraft have been used to determine the position of the barycenter of Saturn in the International Celestial Reference Frame with an accuracy $\leq 0.2$ mas at each epoch, and thus to improve the accuracy of the planetary ephemeris. The planetary ephemeris, a fundamental tool of observational astronomy, has been developed and improved continuously for centuries. A series of JPL planetary ephemerides have been the basis of the Astronomical Almanac tables since 1984.

The motions of Earth and the inner planets are well coupled dynamically and are well tied to the inertial extragalactic reference frame. In contrast, the gas giant planets are not as tightly linked to the inner solar system and not as well linked to the extragalactic reference frame. Our VLBI observations of the Cassini spacecraft orbiting Saturn are the first good opportunity we have had to obtain high accuracy, long-term data for the motion of any gas giant planet. This will improve the tie between the Saturn ephemeris and the inner solar system ephemeris, and will improve the accuracy of extrapolating Saturn positions into the future. The same observational approach is being applied to the multiple spacecraft currently orbiting Mars, and will be applied to the Juno spacecraft as soon as it enters Jupiter orbit in mid-2016. An improved ephemeris will directly help targeting of flyby, aerobreaking, atmospheric entry, and gravity mapping trajectories for future missions. 


\section{Introduction}

The planetary ephemeris is an essential tool for interplanetary spacecraft navigation, as well as the prediction of occultations, tests of general relativity, and other studies of solar system dynamics (including, for example, barycenter corrections for pulsar timing ephemeredes and dynamical mass determinations of solar system bodies). During the past several decades a series of increasingly accurate ephemerides have been developed at JPL by adding new data types such as Very Large Array (VLA) astrometry, spacecraft tracking, and radar range measurements to optical observations (astrolabe, transit and occultation timing, and CCD measurements). The maintenance and improvement of the ephemeris has been a long-term activity of the astronomical community. The accuracy of an ephemeris degrades when extrapolated into the future (or distant past). The rate at which accuracy is lost depends not only on the quality of measurements used to produce a given ephemeris, but the time span of the best observations as well. Our multi-year series of high-accuracy position measurements of the Cassini spacecraft are taking advantage of the extended spacecraft operation provided by the Cassini Solstice Mission. Previous work with Cassini has demonstrated a reduction in ephemeris residuals for Saturn of more than a factor of three.

We also plan new observations of spacecraft orbiting Mars to improve the orbit plane solution via more accurate declinations. In addition, the Juno spacecraft will provide a new opportunity for accurate position measurements of Jupiter after it begins its one-year orbital mission in July 2016. The remaining lifetime of the Cassini mission and the approved Juno mission provide a unique opportunity, not likely to occur again for decades, to obtain accurate long-term positional data for the two planets that dominate the dynamics of our solar system. By combining observations made with radio interferometry (using the Very Long Baseline Array) and spacecraft orbit solutions from Deep Space Network (DSN) tracking, the Saturn, Mars, and Jupiter barycenter positions can be determined with an accuracy of one nano-radian (0.2 milli-arcsecond) at each epoch. This corresponds to about $1.5 \mathrm{~km}$ at the average distance of Saturn, and less than 100 meters at the closest distance of Mars.

Accurate ephemerides are one of the basic tools of observational astronomy, in the same sense as star catalogs and redshift surveys. They represent a community resource whose value is proportional to their accuracy, and whose accuracy requires regular observational support to maintain and improve. A specific example is the great improvement between timing distances and kinematic distances for pulsars when using the newer DE405 ephemeris [1] compared with the older DE200 ephemeris [2]. An improved ephemeris will benefit future planetary missions by reducing the fuel needed for late trajectory corrections, thereby reducing mission risk and leaving more mass available for instruments or for reducing spacecraft mass and cost.

The Pioneer and Voyager missions provided essentially single data points during their flybys of the outer planets, and the Galileo mission to Jupiter was severely constrained by the loss of high gain antenna. There have been VLBI observations of Galileo, but with typical accuracies of only $\sim 10$ mas. Thus, Cassini has been our first opportunity to incorporate highaccuracy data from a spacecraft orbiting an outer planet for an extended period of time, and 
Juno will be our first opportunity to acquire high-accuracy data from a spacecraft orbiting the most massive planet. Our goal is to improve the accuracy of barycenter position measurements of Saturn, Mars, and Jupiter in the inertial International Celestial Reference Frame (ICRF, see [3]; [4]) through phase-referenced very-long-baseline interferometery (VLBI) observations of Cassini, multiple Mars orbiters, and eventually Juno using their downlink signals at $8.4 \mathrm{GHz}$.

By extending our VLBI observations to 2016 we will have high accuracy measurements over more than a quarter of Saturn's orbital period. The error in determining the plane of Saturn's orbit (latitude) decreases rapidly as the time span of observations exceeds $1 / 4$ of the orbital period. The error in longitude decreases approximately linearly with time span. For Mars, the greatest ephemeris uncertainly is the orbit plane. This can be rapidly improved with a short series of new VLBA observations of the Mars Express, Mars Odyssey, and Mars Reconnaissance Orbiter spacecraft.

\section{Observations and Analysis}

The VLBA has demonstrated an unrivaled astrometric precision of less than 10 microarcsec in particularly favorable circumstances [5], and is an ideal instrument for this work due to its high stability, calibration quality, and good coverage of all ecliptic declinations.. We use standard VLBA phase-referencing techniques with rapidly alternating scans between Cassini and angularly nearby reference sources. Note that by scheduling some of our epochs near the times when Saturn reverses direction on the sky we can use the same calibration source for multiple epochs. This minimizes some potential errors. Calibration, editing, and other data reduction tasks are carried out using the Astronomical Image Processing System (AIPS).

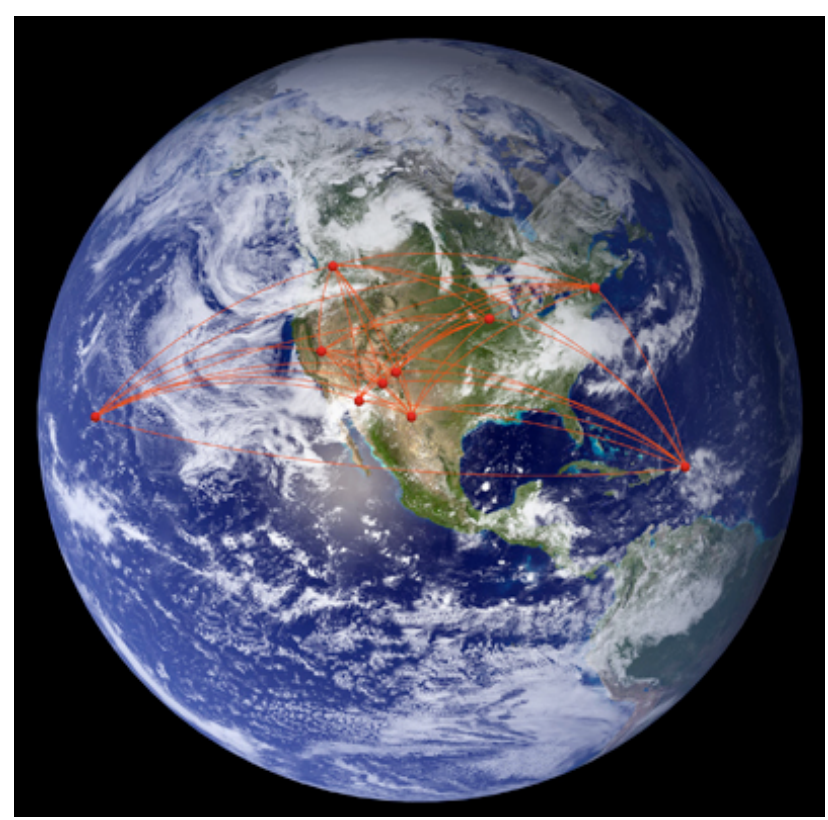

Figure 1: Location of the ten VLBA antennas. 
Every 14-16 minutes we include a scan on a nearby, stronger source from the ICRF catalog to tie our weaker phase reference calibrator and Cassini directly to the ICRF. In addition, we observe several strong sources spread over the sky at the start and end of each epoch to allow better calibration of the troposphere ([6]; [7]). Our 4 VLBA observing bands are used to span a frequency range of $500 \mathrm{MHz}$ to produce accurate group delays.

The rotational accuracy of the ICRF is approximately 0.02 mas, based on $\sim 100$ defining sources each with a typical position error of 0.1-0.2 mas. Individual sources may have larger errors at some epochs due to changes in source structure (e.g., [8]), but the imaging capability of the VLBA allows us to detect and correct for such changes. To maximize the accuracy of our link between the Saturn ephemeris and the ICRF we will devote one of our four epochs each year to tying our phase reference sources to 10-20 high-quality ICRF sources. This will reduce our sensitivity to the structure of individual sources. We expect, based on our previous experience with phase referenced VLBI (e.g., [9]), that an absolute accuracy of 0.1 mas with respect to the ICRF can be achieved for our calibration sources. Thus, the combined error of our Cassini positions with respect to the ICRF will be approximately 0.2 mas, consistent with the level of VLBI spacecraft tracking error predicted by [10].

\section{Results}

Following standard phase-referencing procedures, we create images of both the nearby reference source and Cassini, without self-calibration. The critical importance of manual calibration of the troposphere is shown in figure 2. The position of the peak Cassini signal is measured and the image shifted to the nominal phase center. With the high SNR and welldefined centroids in our Cassini images, two-dimensional Gaussian fits to the peaks provide positions relative to the nominal phase center with formal errors well under 0.1 mas. This position shift, plus any residual position offset measured from the small post-shift baseline phases, is used to calculate the total phase delay for Cassini. The total interferometer delay is the observable used by the JPL navigation and ephemeris software.
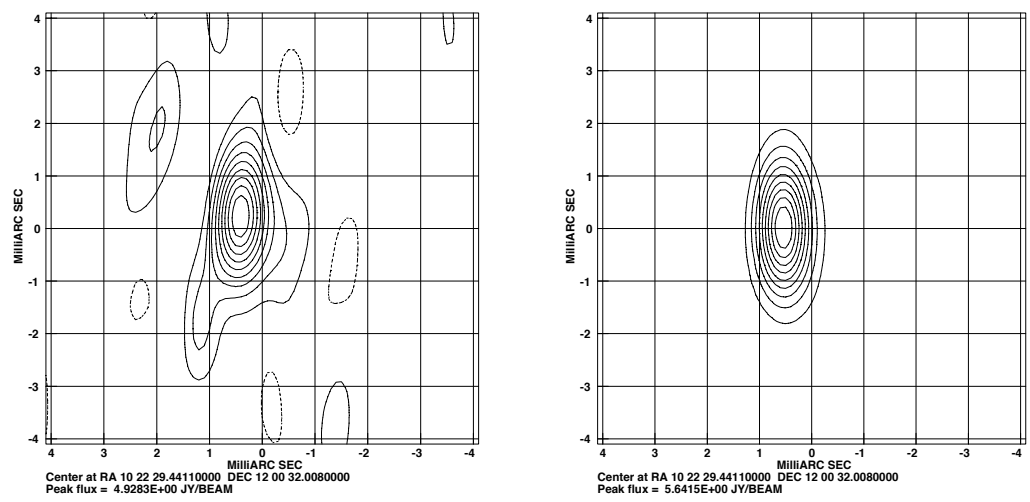

Figure 2: VLBA images of Cassini before (left) and after (right) tropospheric delay calibration. In addition to a noticeable position shift in declination, there is a significant improvement in the quality of the image and an increase in the peak flux density. 
Table 1 lists our current results from VLBA observing epochs through early 2009 [11], and Figure 3 plots the post-fit position residuals from the left two columns in Table 1.

Table 2: Saturn Barycenter Position Residuals (arcsec) for DE405 and DE422 Ephemeris

\begin{tabular}{|c|c|c|c|c|c|}
\hline $\begin{array}{c}\text { Observation } \\
\text { Date }\end{array}$ & Time (TDB) & $\begin{array}{c}\text { RA offset } \\
\text { vs. DE405 }\end{array}$ & $\begin{array}{c}\text { DEC offset } \\
\text { vs. DE405 }\end{array}$ & $\begin{array}{c}\text { RA offset } \\
\text { vs. DE422 }\end{array}$ & $\begin{array}{c}\text { DEC offset } \\
\text { vs. DE422 }\end{array}$ \\
\hline 2004 Sept. & $18: 00: 00$ & 0.1181 & -0.0400 & -0.0018 & -0.0003 \\
\hline 2004 Oct. & $14: 00: 00$ & 0.1293 & -0.0442 & -0.0000 & 0.0001 \\
\hline 2006 Oct. & $17: 00: 00$ & 0.1306 & -0.0451 & 0.0001 & -0.0001 \\
\hline 2007 March & $07: 00: 00$ & 0.1570 & -0.0476 & 0.0001 & 0.0000 \\
\hline 2007 June & $00: 00: 00$ & 0.1357 & -0.0382 & 0.0002 & 0.0001 \\
\hline 2008 Jan. & $10: 00: 00$ & 0.1491 & -0.0479 & 0.0001 & -0.0000 \\
\hline 2008 June & $00: 00: 00$ & 0.1359 & -0.0368 & 0.0001 & -0.0001 \\
\hline 2008 Aug. & $22: 00: 00$ & 0.1271 & -0.0355 & 0.0000 & -0.0001 \\
\hline 2008 Nov. & $17: 00: 00$ & 0.1287 & -0.0367 & 0.0001 & 0.0008 \\
\hline 2009 Feb. & $14: 00: 00$ & 0.1493 & -0.0409 & -0.0001 & 0.0002 \\
\hline 2009 April & $06: 00: 00$ & 0.1479 & -0.0365 & -0.0001 & 0.0005 \\
\hline
\end{tabular}

$\mathrm{TDB}=$ Barycentric Dynamic Time, a general relativistic coordinate time centered on the solar system barycenter.
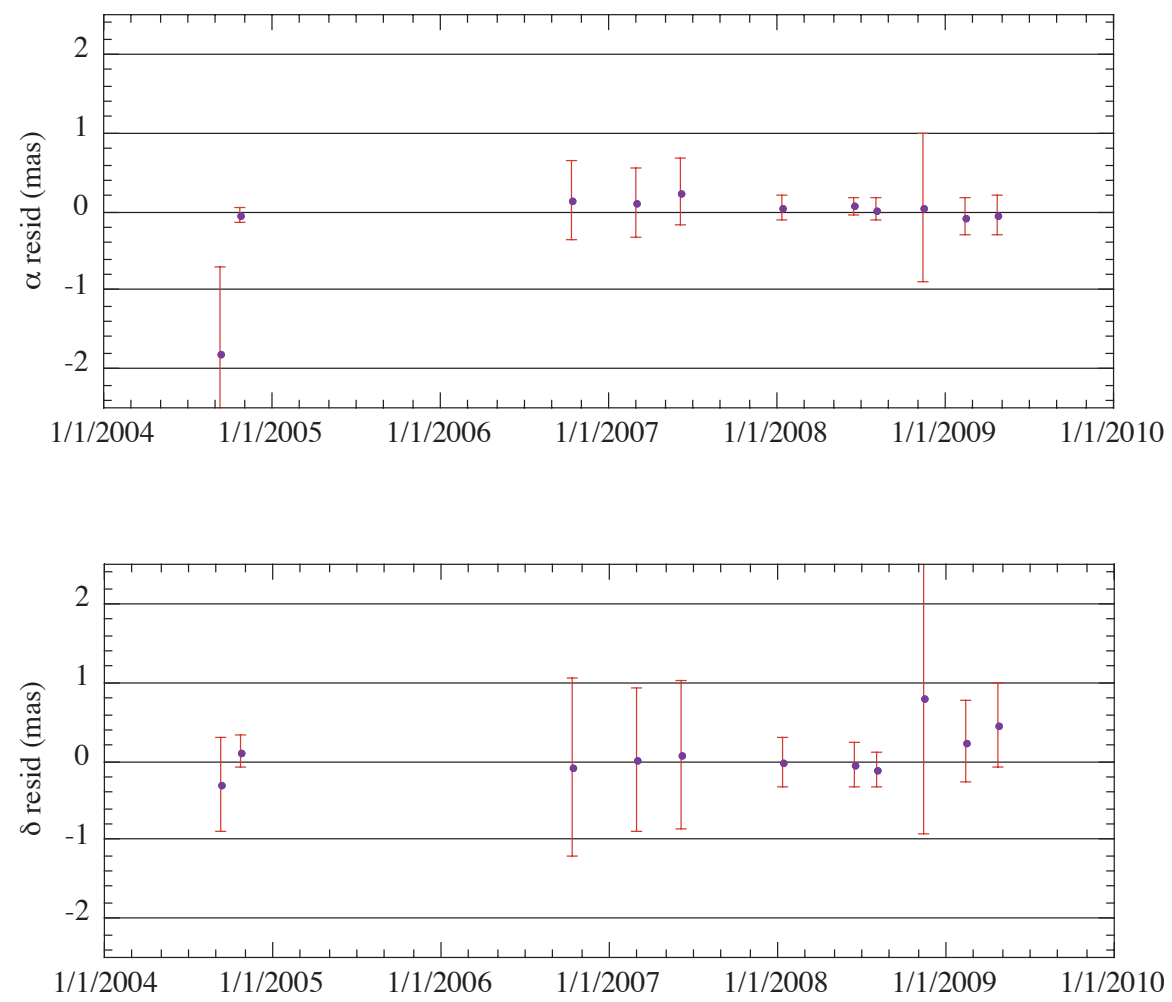

Figure 3: Plot of VLBA Saturn barycenter position residuals after DE422 ephemeris fit. 
The Cassini orbit solutions used to produce Table 1 and Figure 3 were generated by numerical integration of the equations of motion as part of a global Saturn ephemeris and gravity field solution (e.g., [12]; [13]). These solutions are based on a large number of historical and recent observations, and include Newtonian accelerations due to the Sun, planets, and the Saturnian satellites, relativistic perturbations due to the Sun, Jupiter, and Saturn, and perturbations due to Saturn's oblateness. Non-gravitational forces such as spacecraft attitude control, trajectory correction maneuvers, and solar radiation pressure are also modeled.

The DE422 planetary ephemeris is the result of fitting the DE421 ephemeris ([14]; [15]) to the new Cassini VLBA observations along with 18 months of additional tracking data from the Venus Express, Mars Reconnaissance Orbiter, Mars Express, and Mars Odyssey mission and CCD observations of the outer planets. The errors were independently estimated by the ephemeris fitting software at JPL, and include uncertainties in both the VLBA measurements and the positions of Cassini with respect to the Saturn barycenter.

With additional VLBA observations during 2012-2015, the ephemeris uncertainties can be reduced to less than $0.7 \mathrm{~km}$ in longitude, $1.5 \mathrm{~km}$ in latitude, and $0.1 \mathrm{~km}$ in range through at least 2050. This is a factor of 3-5 smaller than the DE421 uncertainty for each coordinate. The Cassini mission will end in 2016, so the next four years are a unique opportunity to obtain the longest VLBA astrometric data set that will possible for any outer planet for decades.

The VLBA is a facility of the National Radio Astronomy Observatory, which is operated by Associated Universities, Inc., under a cooperative agreement with the US National Science Foundation. Part of this research was carried out at the Jet Propulsion Laboratory, California Institute of Technology, under contract with the National Aeronautics \& Space Administration. The JPL authors' copyright for this publication is held by the California Institute of Technology. Government sponsorship acknowledged.

\section{References}

[1] Standish, E. M., 2004, A\&A, 417, 1165.

[2] Verbiest, J. P. W., Bailes, M., van Straten, W., Hobbs, G. B., Edwards, R. T., Manchester, R.N., Bhat, N. D. R., Sarkissian, J. M., Jacoby, B. A., and Kulkarni, S. R., 2008, ApJ, 679, 675.

[3] Ma, C., et al., 1998, AJ, 116, 516.

[4] Fey, A. L., Gordon, D., and Jacobs, C. S. (eds.), 2009, Second Realization of the International Celestial Reference Frame by Very Long Baseline Interferometry, IERS Technical Note 35.

[5] Fomalont, E. B., and Kopeikin, S. M., 2003, ApJ, 598, 704.

[6] Lestrade, J.-F., 2004, in The Role of VLBI in Astrophysics, Astrometry and Geodesy, eds. F. Mantovani and A. Kus (Kluwer Academic Publishers, the Netherlands), 383.

[7] Mioduszewski, A. J., and Kogan, L., 2004, AIPS Memo 110 (www.aips.nrao.edu/aipsdoc.html). 
[8] Fey, A.L., Ma, C., Arias, E.F., Charlot, P., Feissel-Vernier, M., Gontier, A.-M., Jacobs, C.S., Li, J., and MacMillian, D.S., 2004, AJ, 127, 3587.

[9] Lestrade, J.-F., Preston, R. A., Jones, D. L., Phillips, R. B., Rogers, A. E. E., Titus, M. A., Rioja, M. J., and Gabuzda, D. C., 1999, A\&A, 344, 1014.

[10] Lanyi, G., Bagri, D. S., and Border, J. S., 2007, Proc. IEEE, 95, 2193.

[11] Jones, D., Fomalont, E., Dhawan, V., Romney, J., Folkner, W., Lanyi, G., Border, J., and Jacobson, R., 2011, AJ, 141, 29.

[12] Antreasian, P. G., et al., 2007, in AIAA/AAS Astrodynamics Specialist Conference, Macinac Is., MI (AIAA: Reston, VA), paper AAS-07-253.

[13] Jacobson, R. A., Antreasian, P. G., Bordi, J. J., Criddle, K. E., Ionasescu, R., Jones, J. B., Mackenzie, R. A., Meek, M. C., Parcher, D., Pelletier, F. J., Owen, W. M., Jr., Roth, D. C., Roundhill, I. M., and Stauch, J. R., 2006, AJ, 132, 2520.

[14] Folkner, W. M., Williams, J. G., and Boggs, D. H., 2008, JPL Memorandum, 343R-08-003, 20.

[15] Folkner, W. M., Williams, J. G., and Boggs, D. H., 2009, JPL Interplanetary Network Progress Report, 42-178 (2009 August 15). 